\title{
Evolution and trends in the adoption of laparoscopic liver resection in Singapore: Analysis of 300 cases
}

Brian K $\underline{\text { Goh }}{ }^{1,2}{ }_{F R C S E d}$, Zhongkai Wang ${ }^{1}$, Ye-Xin $\underline{\text { Koh }}{ }^{1}{ }_{F R C S E d}$, Kai-Inn $\underline{\operatorname{Lim}}^{3}{ }^{3 B B S}$

\begin{abstract}
Introduction: The introduction of laparoscopic surgery has changed abdominal surgery. We evaluated the evolution and changing trends associated with adoption of laparoscopic liver resection (LLR) and the experience of a surgeon without prior LLR experience.

Methods: A retrospective review of 310 patients who underwent LLR performed by a single surgeon from 2011 to 2020 was conducted. Exclusion criteria were patients who underwent laparoscopic liver surgeries such as excision biopsy, local ablation, drainage of abscesses and deroofing of liver cysts. There were 300 cases and the cohort was divided into 5 groups of 60 patients.

Results: There were 288 patients who underwent a totally minimally invasive approach, including 28 robotic-assisted procedures. Open conversion occurred for $13(4.3 \%)$ patients; the conversion rate decreased significantly from $10 \%$ in the initial period to $3.3 \%$ subsequently. There were $83(27.7 \%)$ major resections and $131(43.7 \%)$ resections were performed for tumours in the difficult posterosuperior location. There were $152(50.7 \%)$ patients with previous abdominal surgery, including $52(17.3 \%)$ repeat liver resections for recurrent tumours, and 60 patients had other concomitant operations. According to the Iwate criteria, 135 (44.7\%) were graded as high/expert difficulty. Major morbidity (>grade 3a) occurred in $12(4.0 \%)$ patients and there was no 30-day mortality. Comparison across the 5 patient groups demonstrated a significant trend towards older patients, higher American Society of Anesthesiologists (ASA) score, increasing frequency of LLR with previous abdominal surgery, increasing frequency of portal hypertension and huge tumours, decreasing blood loss and decreasing transfusion rate across the study period. Surgeon experience ( $\leq 60$ cases) and Institut Mutualiste Montsouris (IMM) high grade resections were independent predictors of open conversion. Open conversion was associated with worse perioperative outcomes such as increased blood loss, transfusion rate, morbidity and length of stay.
\end{abstract}

Conclusion: LLR can be safely adopted for resections of all difficulty grades, including major resections and for tumours located in the difficult posterosuperior segments, with a low open conversion rate.

Ann Acad Med Singap 2021;50:742-50

Keywords: Laparoscopic hepatectomy, laparoscopic liver resection, robotic hepatectomy, robotic liver resection, Singapore

\section{INTRODUCTION}

Over the past few decades, the introduction of laparoscopic surgery has been the biggest game changer in abdominal surgery. ${ }^{1}$ However, although the first laparoscopic liver resections (LLR) were reported in the early $1990 \mathrm{~s},{ }^{2,3}$ widespread adoption of LLR was met with initial skepticism due to technical concerns and the fear of uncontrollable intraoperative bleeding. ${ }^{4-7}$
Furthermore, concerns were raised regarding the use of LLR for hepatic malignancies due to the potential for compromise in resection margins and oncological outcomes. ${ }^{89}$ Nonetheless, despite these initial hesitancies, the adoption of LLR has rapidly increased over the past decade $^{10}$ and LLR has become the standard approach today in many specialised liver centres, especially for minor liver resections for tumours in anterolateral

\footnotetext{
${ }^{1}$ Department of Hepatopancreatobiliary and Transplant Surgery, Singapore General Hospital, Singapore

${ }^{2}$ Duke-National University of Singapore Medical School, Singapore

${ }^{3}$ Department of Anaesthesiology, Singapore General Hospital, Singapore

Correspondence: Prof Brian KP Goh, Department of Hepatopancreatobiliary and Transplant Surgery, Singapore General Hospital, 20 College Road, Academia Level 5, Singapore 169856.

Email: brian.goh@singhealth.com.sg; bsgkp@hotmail.com
} 


\section{CLINICAL IMPACT}

\section{What is New}

- Surgeon experience and difficulty level of liver resections predicted the need for conversion to open surgery.

- Laparoscopic liver resection can be safely performed for liver resections of all difficulty levels including resection of tumours located in the posterosuperior liver segments.

\section{Clinical Implications}

- In carefully selected patients, laparoscopic liver resection can be safely performed with a low rate of open conversion.

segments. ${ }^{11,12}$ Several consensus meetings convened by experts and pioneers in LLR over the past decade have provided recommendations for the development and safe dissemination of LLR. ${ }^{12-14}$

The first LLR was performed in Singapore only in $2004 .{ }^{15}$ The uptake of LLR was slow for the next 7-8 years and only a small number of cases were performed annually. ${ }^{15}$ These were mainly limited to minor resections for tumours in the anterolateral segments. ${ }^{15}$ However, in line with global trends, there was a rapid increase in LLR in institutions in Singapore since 2012 , including its use in major hepatectomies. ${ }^{15,16}$ In 2017, the first large series of LLR documenting 195 resections was published. ${ }^{17}$

The primary objective of the current study was to analyse a single surgeon's experience with the adoption of LLR and its evolution. The secondary objective was to determine predictive factors and the impact of open conversion after LLR.

\section{METHODS}

This is a retrospective review of 310 consecutive patients who underwent LLR performed by a single surgeon from 2011 to 2020. The study was approved by the hospital's institution review board and all data were obtained from a prospective surgical database. The inclusion criteria included all patients who underwent LLR. This included conventional multiport LLR, robotic-assisted LLR, hand-assisted LLR and laparoscopic-assisted resections (hybrid, laparoscopic mobilisation and open parenchymal transection). Exclusion criteria were patients who underwent laparoscopic liver surgeries such as excision biopsy, local ablation, drainage of abscesses and deroofing of liver cysts. Similarly, LLR for unique circumstances such as biliary pathology requiring biliary-enteric anastomoses, vascular reconstruction or donor hepatectomies were excluded.

Prior to the study, the surgeon had no prior experience or formalised training in performing LLR. This study represents an updated experience from a previous report ${ }^{18}$ of the surgeon's surgical training and operation technique. ${ }^{18,19}$ All the resections in this series were performed without a proctor. Only cases where the surgeon was the senior surgeon performing most of the procedure or key parts of the procedure were included in the series. Surgeries where the surgeon acted as a tutor or assistant for colleagues were not included. As the hospital is an academic institution, many of the LLR or parts of the procedure can be performed by surgical residents or fellows under direct supervision.

\section{Definitions}

Postoperative morbidity was classified and graded according to the Clavien-Dindo system. ${ }^{20}$ All postoperative morbidity was recorded up to 30 days or during the index hospital stay, with 30-day readmissions also recorded. The totally laparoscopic approach included any procedure that was attempted by conventional laparoscopy, while the robotic-assisted LLR included any procedure whereby the robot was docked. Open conversion was defined as any LLR whereby the preoperative plan was for laparoscopic resection but the procedure had to be completed (for mobilisation or transection) via an open incision regardless of the length of the incision.

The extent and type of liver resections were classified according to the Brisbane classification published in 2000 whereby a major hepatectomy was defined as a resection of $\geq 3$ contiguous segments. ${ }^{21}$ Additionally, as previously reported, ${ }^{18,19,22}$ resections of segments $6 / 7$ (right posterior sectionectomies) and segments 5/8 (right anterior sectionectomies) were considered as technical major hepatectomies. Segments $1 / 4 \mathrm{a} / 7 / 8$ were considered posterosuperior segments whereas segments $2 / 3 / 4 \mathrm{~b} / 5 / 6$ were considered as anterolateral segments. The LLR were also classified according to the Iwate difficulty scoring system ${ }^{23}$ and Institut Mutualiste Montsouris (IMM) system. ${ }^{24}$

\section{Statistical analysis}

LLR was divided into 5 consecutive equal groups of 60 patients. This was based on the findings of previous studies, ${ }^{4,18}$ which reported that the learning curve of 
LLR was approximately 60 cases. All statistical analyses were performed using SPSS Statistics software version 21.0 (IBM Corp, Armonk, US). The Jonckheere-Terpstra test or Mantel Haenszel test were used as appropriate. Univariate analyses were performed using chi-square tests, Fischer's Exact test or Mann-Whitney $\mathrm{U}$ tests. Multivariate analyses were performed on factors that were significant $(P<0.05)$ on univariate analyses. Logistic regression analyses were used to perform multivariate analyses. All statistical tests were 2 -sided and $P<0.05$ was considered statistically significant.

\section{RESULTS}

During the study period, 300 LLR that met the study criteria were included (Table 1). The baseline clinicopathologic features and outcomes of the patients are summarised in Table 2. Seven of 228 patients (3.1\%) with malignant tumours had resection margins $<1 \mathrm{~mm}$. There was no 30 -day mortality but there were 2 in-hospital mortalities. The first mortality occurred in a patient with end-stage renal failure on dialysis and liver cirrhosis who underwent minor LLR for liver cancer. The surgery and initial postoperative recovery were uneventful. However, due to social reasons, the patient could not be discharged and unfortunately acquired nosocomial pneumonia several weeks after surgery and eventually demised from sepsis. The second mortality occurred in an elderly patient with ischaemic heart disease and chronic obstructive lung disease who underwent right posterior sectionectomy for colorectal metastases. The operation time was 300 minutes and the estimated blood loss was $300 \mathrm{~mL}$. Unfortunately, he developed early postoperative cerebrovascular accident with hemiparesis. He was successfully stabilised but while awaiting placement at a rehabilitation facility, he acquired viral pneumonia and demised from respiratory failure despite ventilatory support.

\section{Evolution and changing trends across the study period}

Across the study period, we observed significant changes in the baseline clinicopathological features and perioperative outcomes of the patients. Patients were significantly older, of higher American Society of Anesthesiologists (ASA) score, were more likely to have previous abdominal surgery, more likely to undergo totally minimally invasive surgical procedure, more likely to have huge ( $\geq 90 \mathrm{~mm})$ tumours and have an increasing incidence of portal hypertension over the study period. This was also associated with a significant decrease in median blood loss and blood transfusion rate over time.

\section{Predictors and outcomes of open conversion}

Thirteen patients experienced open conversion and this was for bleeding $(n=6)$, tumour extent and unclear margins $(n=2)$, slow progress $(n=2)$, difficulty localising tumour $(n=1)$ and dense adhesions $(n=2)$ (Table 3$)$. Only surgeon experience ( $\leq 60$ cases) and high grade were independent predictors of open conversion on multivariate analyses. Patients who underwent open conversion had significantly increased blood loss, transfusion rate, postoperative morbidity and major morbidity, and postoperative length of stay.

\section{DISCUSSION}

We report in this study a single surgeon's experience with the adoption of LLR over the past decade. Over time, LLR was performed on significantly higher risk patients requiring technically more demanding resections (older age, higher ASA score, increased frequency of portal hypertension, increased frequency of huge tumours, and increased frequency of patients with previous abdominal surgery). Despite this, there was a significant decrease in the open conversion rate, blood loss and blood transfusion rate.

Interestingly, it is worth highlighting that the proportion of high difficulty cases did not change significantly over time. During the first 60 cases, $45 \%$ of the cases were graded according to the Iwate criteria as high or expert level, and $38 \%$ of the cases were performed for tumours located in the difficult posterosuperior segments. However, the open conversion rate was only $10 \%$. This was possible as we had utilised the hand-assisted or laparoscopic-assisted (hybrid) technique in 9 cases $(15 \%)$ during our early experience, enabling us to perform complicated resections successfully early on in our learning curve. In our opinion, the hand-assisted and hybrid approaches enabled us to embark safely on difficult LLR and were extremely useful as an initial stepping stone towards performing difficult hepatectomies fully laparoscopically. ${ }^{18}$

A major obstacle to the widespread dissemination of LLR is the long steep learning curve reported by pioneering surgeons. In a landmark paper from France in $2009{ }^{4}$ Vigano et al. reported that the learning curve of minor LLR in terms of open conversion rate was 60 cases. Open conversion rates for minor LLR decreased from $15.5 \%$ to $10.5 \%$ after approximately 60 cases and further decreased to $3.4 \%$ after 116 cases. This long steep learning curve was similarly reported by other pioneer surgeons. ${ }^{25}$ In Japan, Hasegawa et al. reported their institution learning experience with 245 LLR. ${ }^{5}$ They revealed that major hepatectomies could 
Table 1. Types of laparoscopic liver resection in 300 patients

\begin{tabular}{lc}
\hline Resection type & No. (\%) \\
\hline Minor hepatectomy $(\mathrm{n}=218)$ & $75(25.0)$ \\
Wedge resection - anterolateral segments & $30(10.0)$ \\
Wedge resection - posterosuperior segments & $41(13.7)$ \\
Left lateral sectionectomy & $43(14.3)$ \\
Segmentectomy/bisegmentectomy - anterolateral segments & $29(9.7)$ \\
Segmentectomy/bisegmentectomy - posterosuperior segments & $27(9.0)$ \\
Major hepatectomy (n=82) & $8(2.7)$ \\
Right posterior sectionectomy (segment 6/7) & $13(4.3)$ \\
Right anterior sectionectomy (segment 5/8) & $3(1.0)$ \\
Left hepatectomy \pm caudate & $23(7.7)$ \\
Extended left hepatectomy \pm caudate & $1(0.3)$ \\
Right hepatectomy \pm caudate & $7(2.3)$ \\
Extended right hepatectomy \pm caudate & \\
Central hepatectomy & \\
\hline
\end{tabular}

be attempted safely only after having performing 64 minor hepatectomies. This stepwise approach resulted in excellent outcomes associated with the 44 laparoscopic major hepatectomies performed subsequently with an open conversion rate of $6.8 \%$ and low major morbidity rate of $13.6 \%$. Concordant with these findings, Nomi et al., who used cumulative sum analysis, reported that the single surgeon learning curve of a pioneering surgeon in terms of operation time was $45-75$ cases for major hepatectomies, even after the surgeon had acquired sufficient experience with minor hepatectomies. ${ }^{26}$

More recently, a multicentre European study compared the learning curve according to length of stay between 4 pioneering surgeons in stage 2 , with 4 early adopting surgeons in stage 3 of the IDEAL (idea, development, exploration, assessment and long-term follow-up) framework. ${ }^{27}$ The study reported that the early adopting surgeon could achieve comparable outcomes after only 46 cases compared to the pioneering surgeon's 150 cases. ${ }^{27}$ Similarly, the findings of a recent systematic review analysing the learning curves of LLR supported these findings and reported that the number of cases needed to surmount the learning curve of LLR steadily decreased over time. ${ }^{28}$ The study also suggested that the learning curve of robotic-assisted liver resections seemed to be shorter than conventional LLR.

The findings of the present study similarly indirectly support the observations of these recent studies that the learning curve for the modern-day surgeon is much shorter than that of pioneering surgeons. ${ }^{18,27,28}$ Despite a high proportion of patients requiring complicated resections such as resections with a high difficulty score, ${ }^{29}$ major LLR, ${ }^{30}$ repeat LLR, ${ }^{19}$ cirrhosis $^{31}$ and huge tumours $^{32}$ in our early experience, the LLR could be completed successfully with a low open conversion rate and low morbidity. It is important to add that in our analysis, we have intentionally included the different approaches for minimally invasive liver resections, including hand-assistance and robotic-assistance, to reflect the real-world situation. ${ }^{18}$ Confining the analysis to a particular approach such as conventional laparoscopy and excluding other cases performed by other minimally invasive surgical approaches may mislead the reader on the number of cases and outcomes during the learning curve.

One of the major concerns with LLR is the occurrence of unplanned open conversions. This is most commonly reported to be due to intraoperative bleeding..$^{33-35}$ During a surgeon's early learning curve, a high open conversion rate especially from bleeding could theoretically negate many of the benefits of LLR and could even result in poorer perioperative outcomes. ${ }^{34,35}$ In our study, surgeon experience and IMM high grade procedures were the only independent predictors of open conversion. Concordant with the findings from previous published studies, ${ }^{33,34}$ we found that the need for open conversion resulted in poorer perioperative outcomes such as increased blood loss, higher transfusion rate, longer operation time, longer postoperative stay and higher postoperative morbidity rate. These findings highlight the importance for surgeons embarking on LLR to select cases with complexities appropriate to their level of experience to minimise the need for open conversion.

In our experience, most of the open conversions for bleeding were due to slow progress and persistent slow oozing without the need for emergency conversion. It is imperative that surgeons embarking on LLR learn the techniques of controlling bleeding intracorporeally (even if temporarily) prior to open conversion. Various techniques such as compression with gauze or other mechanical haemostats, application of clips, suturing, elevation of the liver and finger compression (hand- 


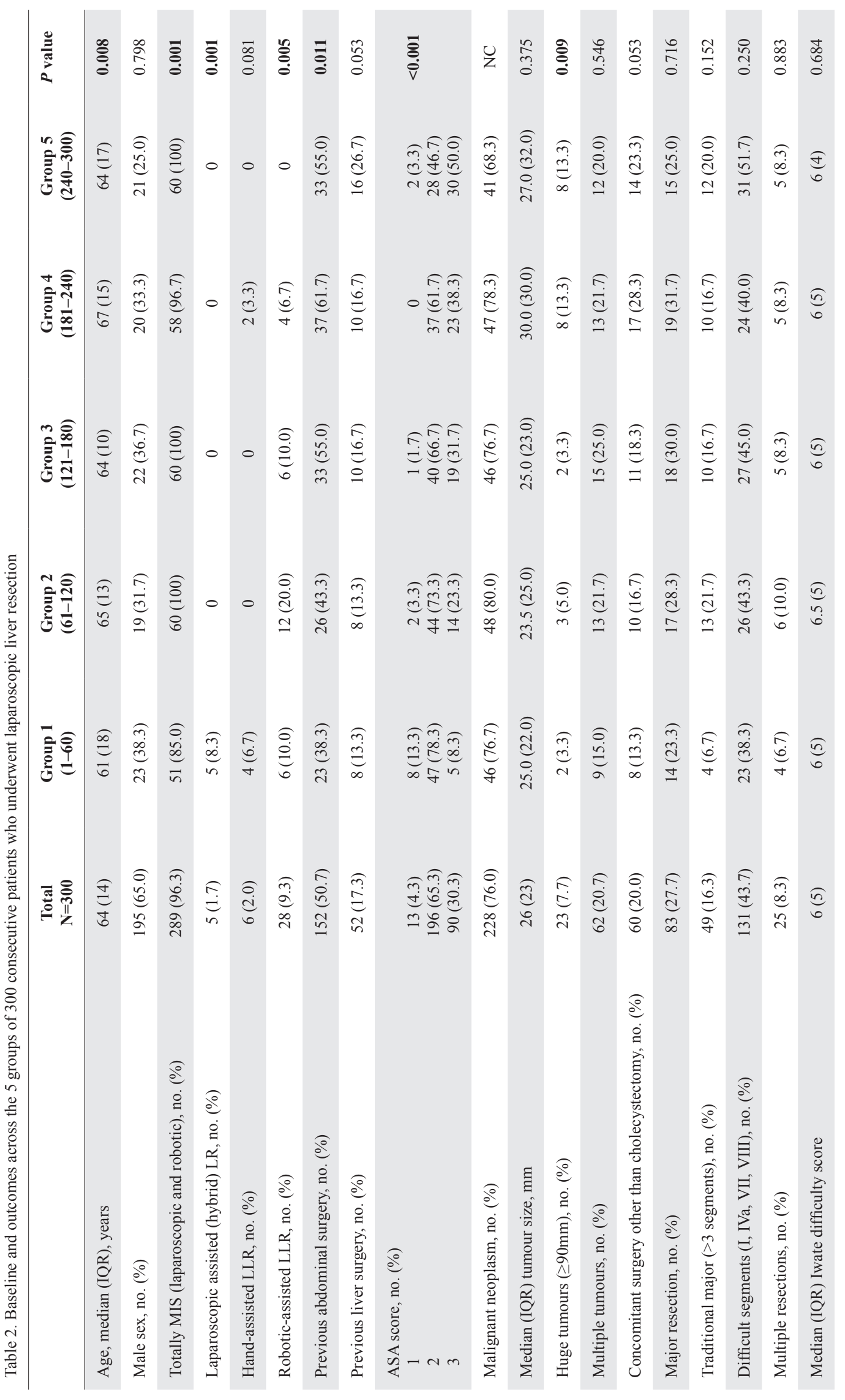




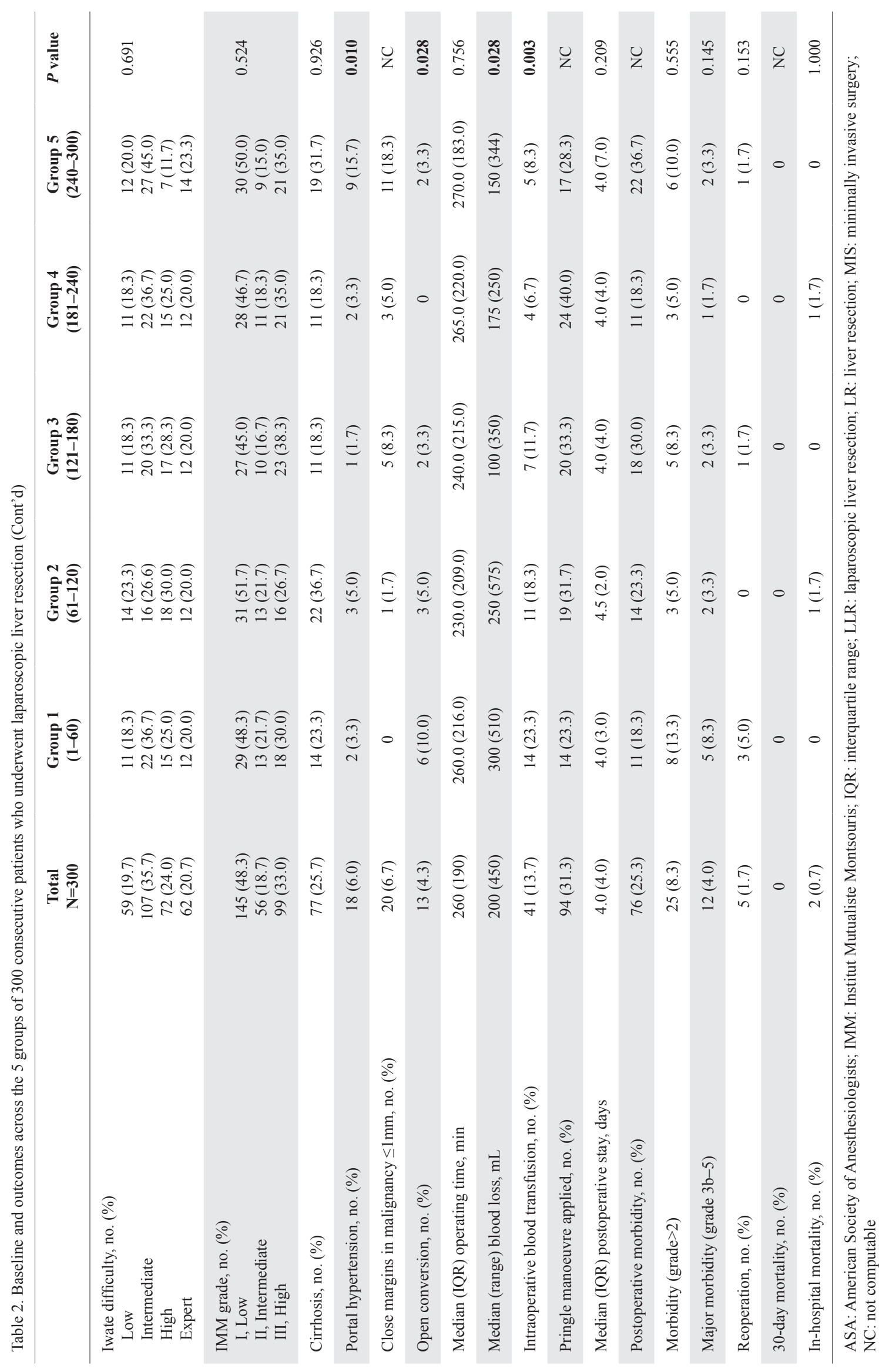


Table 3. Factors associated with and outcomes of open conversion after laparoscopic liver resection in 300 patients

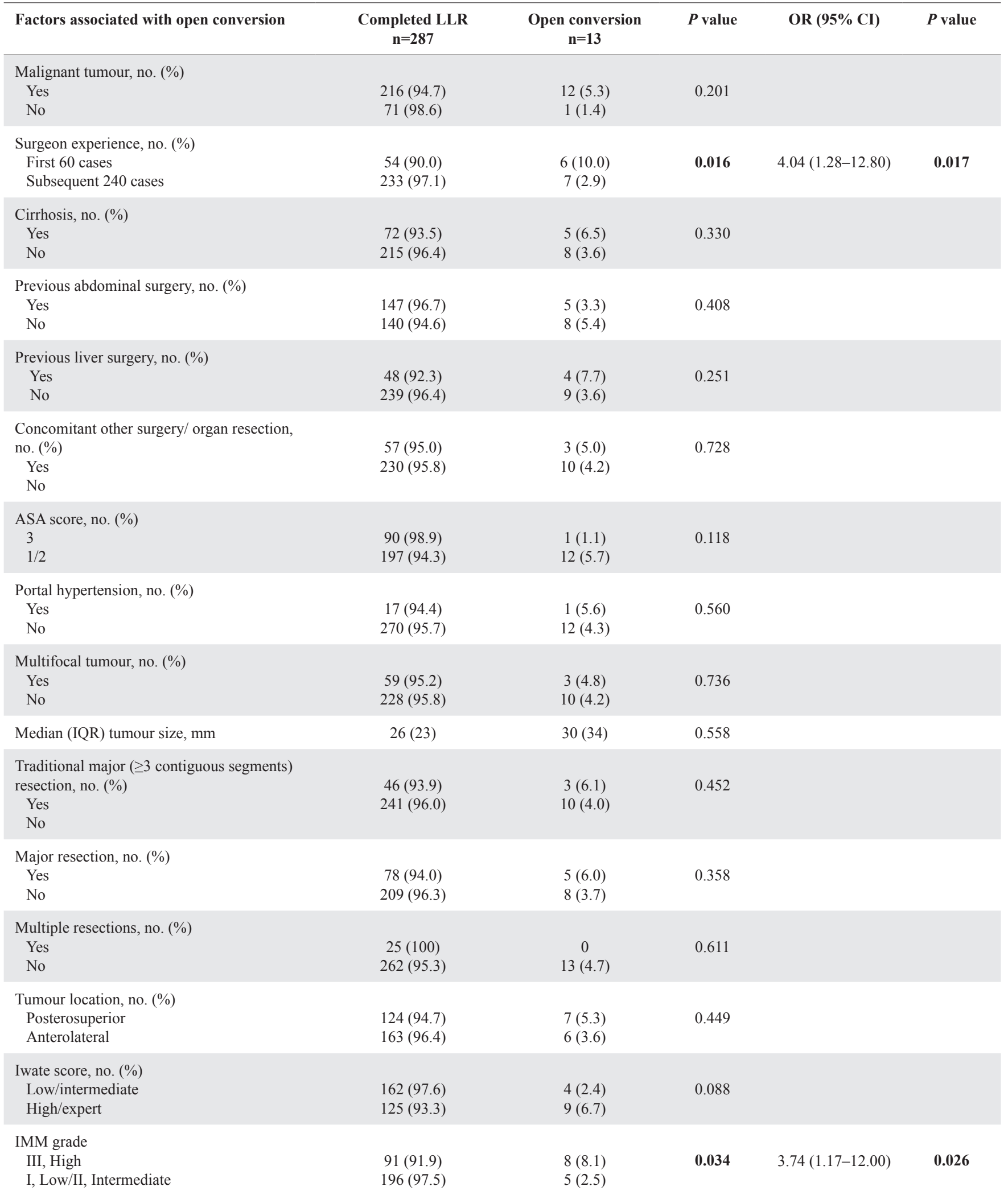


Table 3. Factors associated with and outcomes of open conversion after laparoscopic liver resection in 300 patients (Cont'd)

\begin{tabular}{|c|c|c|c|c|c|}
\hline Factors associated with open conversion & $\begin{array}{l}\text { Completed LLR } \\
\quad \mathbf{n}=\mathbf{2 8 7}\end{array}$ & $\begin{array}{l}\text { Open conversion } \\
n=13\end{array}$ & $P$ value & OR $(95 \% \mathrm{CI})$ & $P$ value \\
\hline \multicolumn{6}{|l|}{ Outcome of open conversion } \\
\hline Median (IQR) operation time, min & $255(190)$ & $405(270)$ & 0.001 & & \\
\hline \multicolumn{6}{|l|}{ Intraoperative blood transfusion, no. (\%) } \\
\hline Yes & $31(10.8)$ & $10(76.9)$ & $<0.001$ & & \\
\hline Median (IQR) estimated blood loss, $\mathrm{mL}$ & $200(350)$ & $1500(1250)$ & $<0.001$ & & \\
\hline $\begin{array}{l}\text { Median (IQR) postoperative hospitalisation, } \\
\text { days }\end{array}$ & $4(3)$ & $8(12)$ & $<0.001$ & & \\
\hline \multicolumn{6}{|l|}{ Postoperative morbidity, no. (\%) } \\
\hline Yes & $65(22.6)$ & $11(84.6)$ & $<0.001$ & & \\
\hline \multicolumn{6}{|l|}{ Major morbidity (grade>2), no. (\%) } \\
\hline Yes & $21(7.3)$ & $9(69.2)$ & 0.021 & & \\
\hline No & $266(92.7)$ & $4(30.8)$ & & & \\
\hline \multicolumn{6}{|l|}{ Mortality, no. (\%) } \\
\hline 30-day & 0 & 0 & 1.000 & & \\
\hline In-hospital & $2(0.7)$ & 0 & & & \\
\hline
\end{tabular}

ASA: American Society of Anesthesiologists; CI: confidence interval; IMM: Institut Mutualiste Montsouris; IQR: interquartile range;

LLR: laparoscopic liver resection; OR: odds ratio

assistance) can be used for haemostasis depending on the situation. ${ }^{33}$ It is important to highlight that surgeons should be prepared to convert early if deemed necessary, especially during their early experience, as this may potentially reduce blood loss and postoperative morbidity. ${ }^{35}$

Due to its retrospective nature, there are various limitations associated with the current study. As previously highlighted, ${ }^{18}$ it is important to emphasise that the surgeon's experience in this study is unique and may not be directly translated to that of other surgeons embarking on LLR. Firstly, the surgeon had a vast prior experience with open and complex hepatopancreatobiliary surgeries, including transplantation before embarking on LLR. Secondly, although the surgeon had no formal training and prior exposure to LLR, he had extensive experience in other complex laparoscopic procedures such as donor nephrectomies. Thirdly, during the study period, the surgeon had also gained further laparoscopic experience by performing other abdominal procedures such as pancreatectomies, gastric resections and major biliary procedures. ${ }^{7,36}$ Fourthly, as the surgeon was practising in a high-volume centre, he had gained further concomitant experience with LLR by tutoring and assisting other surgeons who were adopting LLR during the study period. Finally, it is also important to add that the learning curve of a surgeon exposed to LLR, such as an LLR fellowship-trained surgeon, would likely be shorter and less steep than the current reported experience.

In conclusion, this study based on a single surgeon's experience demonstrates that LLR can be safely adopted for resections of all difficulty grades, including major resections and resection of tumours located in the difficult posterosuperior segments with a low open conversion rate. Surgeon experience and IMM high grade resections were significant factors associated with open conversion.

\section{Disclosure}

Brian $K$ Goh has received honorarium and travel grants from Johnson \& Johnson, Transmedic Pte Ltd, the local distributor for Intuitive Surgical Inc, Medtronic and Olympus Singapore.

\section{REFERENCES}

1. Goh BK, Teo RY. Current status of laparoscopic and robotic pancreatic surgery and its adoption in Singapore. Ann Acad Med Singap 2020;49:377-83.

2. Reich H, McGlynn F, DeCaprio J, et al. Laparoscopic excision of benign liver lesions. Obstet Gynecol 1991;78:956-8. 
3. Gagner M, Rheault M, Dubuc J. Laparoscopic partial hepatectomy for liver tumor. Abstracts of the 1992 scientific session of the Society of American Gastrointestinal Surgeons (SAGES), Washington DC, USA. 1992:85-110.

4. Vigano L, Laurent A, Tayar C, et al. The learning curve in laparoscopic liver resection: improved feasibility and reproducibility. Ann Surg 2009;250:772-82.

5. Hasegawa Y, Nitta H, Takahara T, et al. Safely extending the indications of laparoscopic liver resection: when should we start laparoscopic major hepatectomy? Surg Endosc 2017;31:309-16.

6. Tomassini F, Scuderi V, Colman R, et al. The single surgeon learning curve of laparoscopic liver resection. A continuous evolving process through stepwise difficulties. Medicine (Baltimore) 2016;95:e5138.

7. Goh BK, Low TY, Teo JY, et al. Adoption of robotic liver, pancreatic and biliary surgery in Singapore: a single institution experience with its first 100 consecutive cases. Ann Acad Med Singap 2020;49:742-8.

8. Syn N, Kabir T, Koh YX, et al. Survival advantage of laparoscopic versus open resection for colorectal liver metastases: a meta-analysis of individual patient data from randomized trials and propensity-score matched studies. Ann Surg 2020;272:253-65.

9. Chang SK, Tay CW, Shen L, et al. Long-term oncological safety of minimally invasive hepatectomy in patients with hepatocellular carcinoma: a case-control study. Ann Acad Med Singap 2016;45:91-7.

10. Ciria R, Cherqui D, Geller DA, et al. Comparative short-term benefits of laparoscopic liver resection: 9000 cases and climbing. Ann Surg 2016;263:761-77.

11. Aghayan DL, Kazaryan AM, Fretland ÅA, et al. Evolution of laparoscopic liver surgery: 20-year experience of a Norwegian high-volume referral center. Surg Endosc 2021. doi:10.1007/s00464021-08570-3.

12. Wakabayashi G, Cherqui D, Geller DA, et al. Recommendations for laparoscopic liver resection: a report from the second international consensus conference held in Morioka. Ann Surg 2015;261:619-29.

13. Buell JF, Cherqui D, Geller DA, et al. The international position on laparoscopic liver surgery: The Louisville Statement, 2008. Ann Surg 2009;250:825-30.

14. Abu Hilal M, Aldrighetti L, Dagher I, et al. The Southampton consensus guidelines for laparoscopic liver surgery: From indication to implementation. Ann Surg 2018;268:11-8.

15. Tan $\mathrm{CH}$, Tan $\mathrm{KH}$, Lee $\mathrm{CL}$, et al. Observing an upward trajectory in minimally invasive hepatectomies in Singapore-a nationwide analysis. Ann Laparosc Endosc Surg 2018;3:78.

16. Goh BKP, Lee SY, Teo JY, et al. Changing trends and outcomes associated with the adoption of minimally invasive hepatectomy: a contemporary single-institution experience with 400 consecutive resections. Surg Endosc 2018;32:4658-65.

17. Goh BK, Teo JY, Chan CY, et al. Evolution of laparoscopic liver resection at Singapore General Hospital: a nine-year experience of 195 consecutive resections. Singapore Med J 2017;58:708-13.

18. Goh BKP, Prieto M, Syn N, et al. Critical appraisal of the learning curve of minimally-invasive hepatectomy: experience with the first 200 cases of a Southeast Asian early adopter. ANZ J Surg 2020; 90:1092-98.

19. Mohan R, Kabir T, Wu AGR, et al. Analysis of perioperative outcomes following laparoscopic repeat liver resection compared to laparoscopic primary liver resection based on a single surgeon's experience: a 1:2 propensity score-matched study. Surg Oncol 2020;35:382-7.

20. Dindo D, Demartines N, Clavien PA. Classification of surgical complications: a new proposal with evaluation in a cohort of 6336 patients and results of a survey. Ann Surg 2004;240:205-13.

21. Strasberg SM. Nomenclature of hepatic anatomy and resection: a review of the Brisbane 2000 system. J Hepatobiliary Pancreat Surg 2005;12:351-5.

22. Hwang DM, Han HS, Yoon YS, et al. Laparoscopic major liver resection in Korea: a multicenter study. J Hepatobiliary Pancreat Sci 2013;20:125-30.

23. Wakabayashi G. What has changed after the Morioka consensus conference 2014 on laparoscopic liver resection. Hepatobiliary Surg Nutr 2016;5:281-9.

24. Kawaguchi Y, Fuks D, Kokudo N, et al. Difficulty of laparoscopic liver resection: proposal for a new classification. Ann Surg 2018;267:13-7.

25. Komatsu S, Scatton O, Goumard C, et al. Development process and technical aspects of laparoscopic hepatectomy: learning curve based on 15 years experience. J Am Coll Surg 2017;224:841-50.

26. Nomi T, Fuks D, Kawaguchi Y, et al. Learning curve for laparoscopic major hepatectomy. Br J Surg 2015;102:796-804.

27. Halls MC, Alseidi A, Berardi G, et al. A comparison of the learning curves of laparoscopic liver surgeons in differing stages of the IDEAL paradigm of surgical innovation. Ann Surg 2019;269:221-8.

28. Chua D, Syn N, Koh YX, et al. Learning curves in minimally invasive hepatectomy: systematic review and meta-regression analysis. Br J Surg 2021;108:351-8.

29. Goh BKP, Prieto M, Syn N, et al. Validation and comparison of the Iwate, IMM, Southampton and Hasegawa difficulty scoring systems for primary laparoscopic hepatectomies. HPB (Oxford) 2021;23:770-6.

30. Goh BKP, Lee SY, Koh YX, et al. Minimally invasive major hepatectomies: a Southeast Asia single institution contemporary experience with its first 120 consecutive cases. ANZ J Surg 2020;90:553-7.

31. Goh BK, Syn N, Lee SY, et al. Impact of liver cirrhosis on the difficulty of minimally-invasive liver resections: a 1:1 coarsened exact-matched controlled study. Surg Endosc 2021;35:5231-8.

32. Kabir T, Syn NL, Guo Y, et al. Laparoscopic liver resection for huge $(\geq 10 \mathrm{~cm})$ hepatocellular carcinoma: A coarsened exact-matched single-surgeon study. Surg Oncol 2021;37:101569.

33. Goh BK, Chan CY, Wong JS, et al. Factors associated with and outcomes of open conversion after laparoscopic minor hepatectomy. Surg Endosc 2015;29:2636-42.

34. Troisi RI, Montalti R, Van Limmen JG, et al. Risk factors and management of conversions to an open approach in laparoscopic liver resection: analysis of 265 consecutive cases. HPB (Oxford) 2014; 16:75-82.

35. Costi R, Scatton O, Haddad L, et al. Lessons learned from the first 100 laparoscopic liver resections: not delaying conversion may allow reduced blood loss and operative time. J Laparoendosc Adv Surg Tech A 2012;22:425-31.

36. Goh BKP, Zeng G, Low TY, et al. Changing trends and outcomes associated with the adoption of minimally-invasive pancreato-biliary surgery: contemporary experience of a 'self-taught' early adopter in Southeast Asia. J Minim Access Surg 2020;16:341-7. 